\title{
Electromagnetic Field Analysis of the End Region of a Large Rotating Machine using Nonconforming Mesh Connection
}

\author{
Masafumi Fujita*a) Senior Member, Takahiro Sato* Member, Norio Takahashi* Member, \\ Masashi Kobayashi* Member, Ken Nagakura* Member, Hideyuki Nakamura* Member, \\ Hiroyuki Kaimori** ${ }^{*}$ Member
}

(Manuscript received Jan. 29, 2021, revised Jul. 10, 2021)

\begin{abstract}
In the characteristic evaluations of large rotating machines, such as turbine generators, modeling of complex structures, smallsized parts, or local physical behaviors may lead to ill-conditioned and/or a large number of elements. In this study, analysis modeling for evaluation of the electromagnetic behaviors using the nonconforming mesh connection technique is developed by improving the sliding method to simulate movements and the developed method is applied to model the coil end part of a turbine generator. The analysis results are verified by measurements and with the results of a model without the nonconforming mesh connection. Then, the merits and important points regarding the application of the nonconforming mesh connection technique are described.
\end{abstract}

Keywords : end region, finite element method, nonconforming mesh connection, rotating machine

\section{Introduction}

It is important to evaluate the electromagnetic behaviors in the end region of large rotating machines such as turbine generators because the leakage flux causes eddy current loss ${ }^{(2)-(5)}$. To reduce the eddy current loss in metal structures such as stator core end, end flange and shield plate, it is required to quantify losses precisely. In order to enhance the accuracy of loss prediction, more detailed modeling is required but the modeling of the complex structures, such as the coil end parts and the slits in the tooth of the core plate, is normally laborious. Furthermore, in comparison with the large equipment size, the thickness of the laminated core plates and the strands of the coil are very small and the skin depth is generally thin in the magnetic structures such as end flange. These lead to illconditioned and/or a large number of elements. Then, large scale analysis of turbine generators, including the coil end region and the detailed part of the structures except the local model for each target of evaluation, is difficult to perform and not so many examples have been reported.

Among the many works on the modeling of the end region of rotating machines, a method to project the coil end region partially to the conical coordinate system has been proposed ${ }^{(6)}$ but some of the large rotating machines incorporate end flange outside the coil end which makes it difficult to use conical coordinate transformation. Moreover, 2 potential method ${ }^{(7)}$, in which the current source is defined independently from the finite element

This paper is based on Reference (1), which published in the International Conference on Electrical Machines and Systems (2020) $(2020$ IEEJ

a) Correspondence to: Masafumi Fujita.

E-mail: masafumi1.fujita@toshiba.co.jp

* Toshiba Energy Systems \& Solutions Corporation

2-4, Suehiro-cho, Tsurumi-ku, Yokohama 230-0045, Japan

** Science Solutions International Laboratory, Inc.

2-21-7, Naka-cho, Meguro-ku, Tokyo 153-0065, Japan method (FEM) mesh region, could ease the meshing process but it may take much time to calculate the potential on the boundary surface between the total and reduced potential regions and is not suitable to calculate the eddy currents in the conductors of current source. On the other hand, quality of the meshes generated by automesh techniques has been improved recently but it may sometimes produce too many elements or distorted meshes unexpectedly especially for meshing the coil end and the slits in the tooth, which would lead to low accuracy.

Another approach is nonconforming mesh connection techniques. Some of them are developed to simulate the rotational movement of rotating machines ${ }^{(8)-(11)}$ and some of them are used to reduce the analysis scale eliminating the elements where the fine meshes are not required ${ }^{(12)}$. In (13), the eddy currents in the coil conductor strands of a turbine generator are calculated applying the nonconforming surface ${ }^{(8)}$ to the area between the coil end bars but the detail of the modeling, especially treatment of the core and the connection part is not presented.

In this paper, a nonconforming mesh connection technique, originally developed as the sliding method to simulate the rotational movements of the rotating machines, is improved for the modeling of the coil end part. The conventional method has had constraints that the edges of the elements facing the nonconforming surface form parallel lines in the moving direction so as to minimize the search process of the corresponding edges with one dimensional approach ${ }^{(8)}$. First, this method has been improved to be applicable to local region. Then, it has also been expanded to two dimensional interpolation surface to allow more degrees of freedom to the meshing process. The developed method is applied in the space around the complex shaped coil end bars and verified. Then, the merits and important points regarding the application of the nonconforming mesh connection technique are described. 


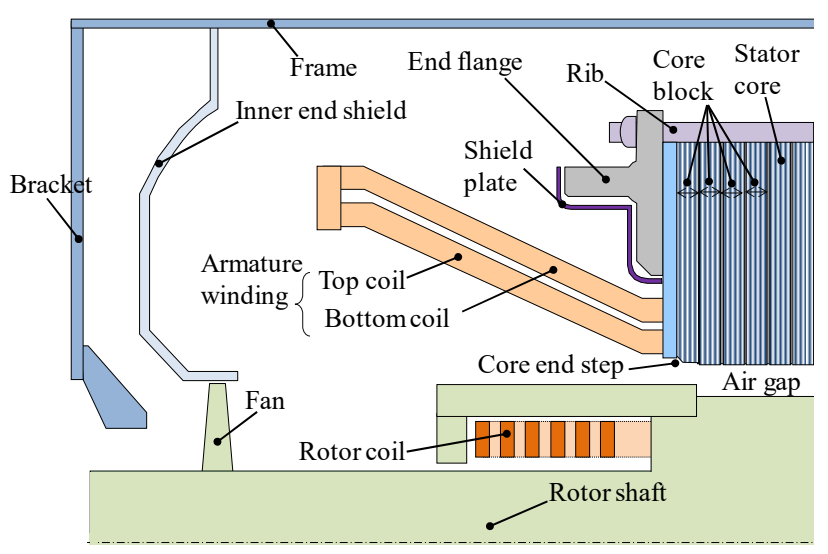

Fig. 1. Schematic view of end region of turbine

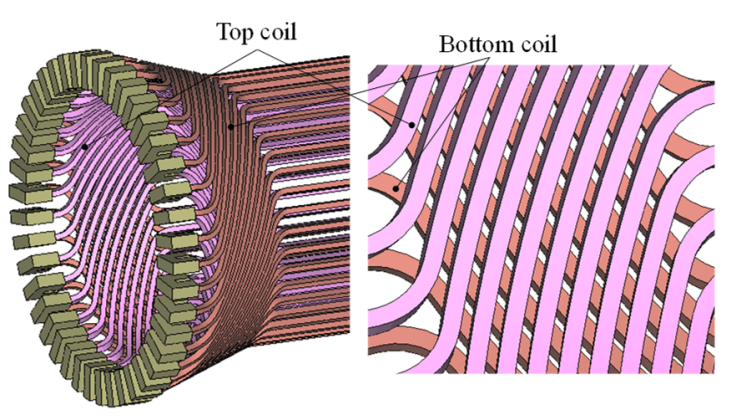

Fig. 2. Stator coil end.

\section{End region of rotating machine}

Figure 1 illustrates an example of the end region of large capacity rotating machines such as a turbine generator ${ }^{(2)}$. The armature winding generally consists of double layers of conducting bars, which are top coil and bottom coil. These conducting bars are bended at the end portion and connected to the corresponding bars with a certain coil pitch. The end part of the coil normally forms a cone and the top coils and the bottom coils are arranged so as to cross in a grid shape shown in Fig. 2.

The stator core is composed of laminated iron plates whose thickness is usually 0.35 or $0.5 \mathrm{~mm}$, which are firmly clamped with the core end flange and the rib in the outer region, and it is installed inside the cylindrical frame. As the core end flange is usually electrically conductive, the copper-made shield plate is settled on the surface of the core end flange for large capacity machines to reduce eddy current loss.

As for the ventilation, a flow of the cooling gas is generated by the fan, mounted in the end region of the shaft, and the gas circulates inside the machine. As for the stator core, ventilation ducts are formed by inserting the inside space blocks in the core with a certain axial distance and the gas flows to cool the coils and the core.

In the end region of the stator core, the core end steps and core end slits are often provided to reduce the eddy current loss by the impinging end flux.

\section{Analysis method}

3.1 Electromagnetic analysis The electromagnetic field is solved by the FEM with edge elements using the magnetic vector potential and the electric scalar potential ${ }^{(14)}$. The matrix is solved by ICCG method.

3.2 Nonconforming mesh connection The analysis method using the nonconforming surface is essentially based on the interpolation for the sliding surface of the moving mesh model ${ }^{(8)}$. The reference (8) deals with two regular mesh parts to simulate the stationary and moving parts shown as Fig. 3 (a), where a relative movement between the upper part ('regular mesh 1') and the lower part ('regular mesh 2') is allowed. Then the nonconforming surface is applied between the regular mesh parts and unknowns are set on the edges of the nonconforming surface. Then the tangential component of magnetic vector potential on the nonconforming surface is interpolated with the potentials on the surface of the regular mesh parts.

\subsubsection{Locally applied nonconforming mesh connection}

The nonconforming surface has to spread to the boundary edges of the analysis region to simulate the movement. Because of this, the nonconforming surface had to be applied in the region without conductive and/or magnetic properties. But for the application to the stationary part, the nonconforming mesh connection may be enough for the local part not for the boundary-to-boundary region. First, this method has been improved to be applicable to the local surface of the stationary part as shown in Fig.3 (b), in order to use the nonconforming mesh connection in the minimum extent required.

\subsubsection{D nonconforming mesh connection}

Secondly, the interpolation is expanded from one dimension (1D) to two dimension (2D) to deal with more complex structure. Figure 4 displays conceptual diagrams of the nonconforming mesh connection. The nonconforming surface is determined in a reticular pattern independent from the nodes on the surface of facing meshes of 'regular mesh 1' and 'regular mesh 2'. Though the regular meshes and the nonconforming surface are in contact with each

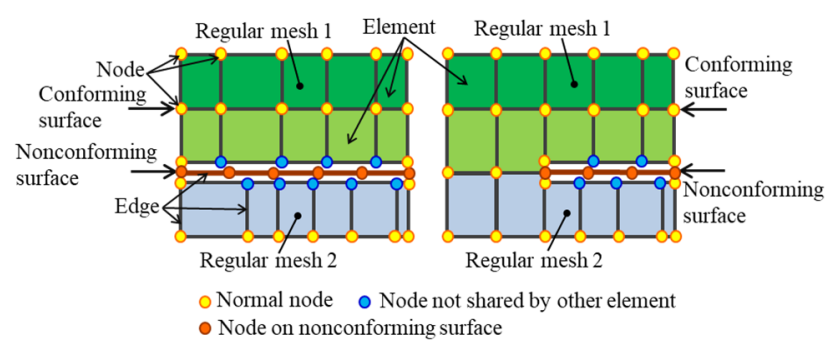

$\begin{array}{ll}\text { (a) Conventional } & \text { (b) Locally applied non- }\end{array}$ sliding surface conforming mesh connection

Fig. 3. Simple description of nonconforming mesh connection.

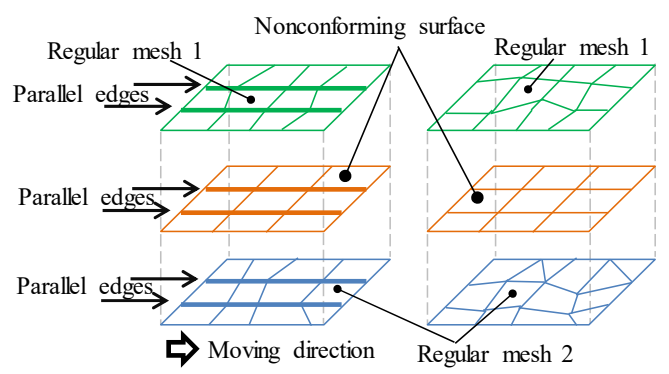

(a) 1D nonconforming mesh connection

(b) $2 \mathrm{D}$ nonconforming mesh connection

Fig. 4. The 1D \& 2D nonconforming mesh connection. 
other, they are depicted separately for explanation in Fig.4.

The 1D nonconforming mesh connection, shown in Fig.4 (a), has constraints that the edges of the elements lie upon the parallel lines in the moving direction (shown as 'parallel edges'). This restriction can minimize the search process of the corresponding edges.

On the other hand, the 2D nonconforming mesh connection, in Fig.4 (b), has no constraints with the nodes or edges and the edges of any triangle and quadrilateral on the facing mesh surface can be interpolated using the edges on the nonconforming surface. As this nonconforming mesh connection does not assume relative movements, the matrix is constructed just once in the preprocessing not for each time step.

To achieve the 2D nonconforming mesh connection, the authors have introduced an interpolation method for the edge crossing multiple rectangular element on the nonconforming surface. In reference (7) and (12), the magnetic vector potential on the edge of the regular mesh $\boldsymbol{A}$ is interpolated using the edge of the nonconforming surface according to;

$$
\boldsymbol{A}=\sum_{i} A_{i} \boldsymbol{N}_{i}
$$

where $A_{i}$ is the magnetic vector potential on the nonconforming surface, $N_{i}$ is the interpolation coefficient determined by the location of the regular mesh and the nonconforming mesh, which uses the coordinates of the start point ' $a$ ' and the end point ' $b$ ' of the edge of the regular mesh.

When the edge of the regular mesh is defined inside the rectangular element composed of the four edges $\left(A_{1}\right.$ to $\left.A_{4}\right)$ as shown in Fig. 5(a), the magnetic vector potential can be interpolated using equation (1). But when the de nse-coarse difference in the mesh is large, which is likely to occur in the large rotating machines described in this paper, the edge of the regular mesh may cross several rectangular elements on the nonconforming surface as illustrated in Fig.5 (b). In this case, the accuracy of the conventional interpolation method might become worse because it uses the edge of the nonconforming surface defined by the coordinate the 2 points ' $a$ ' and ' $b$ ' of the regular mesh. Therefore, in the proposed method, the edge of the regular mesh is divided by each rectangular element of the nonconforming surface and the potential is interpolated for each divided edge. For the configuration of Fig.5 (b), equation (1) can be rewritten as;

$$
\begin{aligned}
\boldsymbol{A} & =\boldsymbol{A}^{(1)}+\boldsymbol{A}^{(2)}+\boldsymbol{A}^{(3)} \\
& =\left(\sum_{i} A_{i} \boldsymbol{N}_{i}\right)^{(1)}+\left(\sum_{j} A_{j} \boldsymbol{N}_{j}\right)^{(2)}+\left(\sum_{k} A_{k} \boldsymbol{N}_{k}\right)^{(3) \cdots \cdots \cdots(}
\end{aligned}
$$

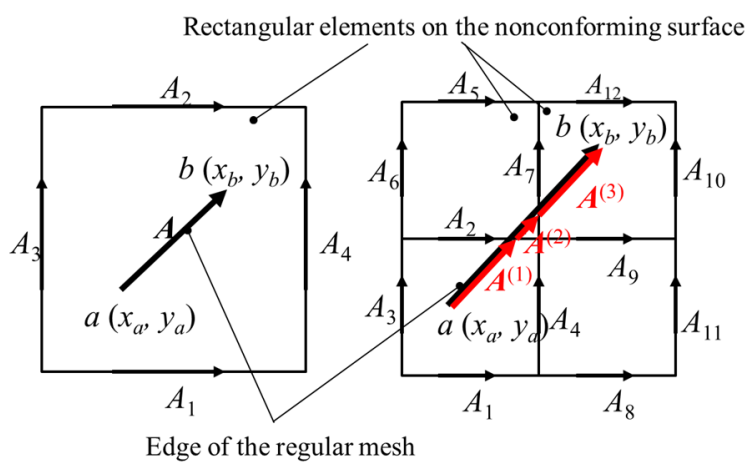

(a) Conventional method

(b) proposed method

Fig. 5. Edge of the regular mesh and the rectangular element of the nonconforming surface.
In this way, the magnetic vector potential can be interpolated for any positional relation between the rectangular element on the nonconforming surface and the edge of the regular mesh, which allows general nonconforming mesh connection.

Though this method enables the facing surface to be free from mesh shape, if the length of the edges facing via a nonconforming surface is extremely different, one edge is required to be interpolated by too many edges and vice versa. The coil end part of large rotating machines such as 2-pole turbine generators, is skewed by much larger angle than, for instance, that of slot skew angle of induction machines and, if the nonconforming mesh connection is applied between the top and bottom coil in the coil end region, a difference of mesh density could lead to the degradation of the analysis accuracy.

\section{Analysis model and modeling process}

4.1 Analyzed machine In this chapter, an analysis model of the end region of a large rotating machine, in which the nonconforming mesh connection - called hereafter 'nonconforming model' (nonconf.)- is applied around the coil end, is described. Here, a $900 \mathrm{MW}$ class 2-pole turbine generator is analyzed and the operation conditions are the open-circuit operation (armature current $=0$ ), the short-circuit operation (terminal voltage $=0$ ), and the loaded operation with leading power factor ${ }^{(15)}$. The end part of the top and bottom coils cross over each other. Furthermore, the end flange and the shield plate, which are conducting parts, require the finer meshes comparing to the equipment size due to the skin effect.

\subsection{Mesh construction}

\subsubsection{Nonconforming model}

To ease the cumbersome meshing process especially for the air region between the solid structures, the finite element meshes are constructed as follows;

i. First, a base plane of 1-pole region (angle of $180^{\circ}$ ) is created in the $\mathrm{x}-\mathrm{y}$ plane shown in Fig. 6.

ii. Meshes on the base plane are extruded in the z-direction as Fig. 7 (a).

iii. The inner coil bar (top coil) and the outer coil bar (bottom coil) are twisted in opposite directions by half an angle of the coil pitch. Then, the top coil and bottom coil are connected by changing the material property of the element corresponding to the connection part in the most end of the coil bar to the conductor as Fig. 7 (b).

iv. The nodes to the coil end part are moved in the radial direction so as to arranged on a cone as Fig. 7 (c).

v. The meshes including the end flange and the shield plate are added outside of the coil end as Fig. 7 (d).

Then the nonconforming surfaces are defined in the air region around the coil end part. Figure 8 and 9 show the nonconforming surfaces (NCS1-3) with a cross-sectional diagram and a bird's-eye view of stator part respectively. The surface 'NCS1' is defined between the air gap and the top coil to enable to ease the extrusion of the stator mesh without constraints due to the rotor part. The surface 'NCS2' between the top coil and the bottom coil is the most effective to ease the constructing the coil end mesh. The surface 'NCS3' between the bottom coil and the shield plate enables the meshes of the conducting parts for the end flange and the shield plate to be well-ordered, otherwise the meshes would be distorted because of the twisted meshes for the bottom coils which may cause 


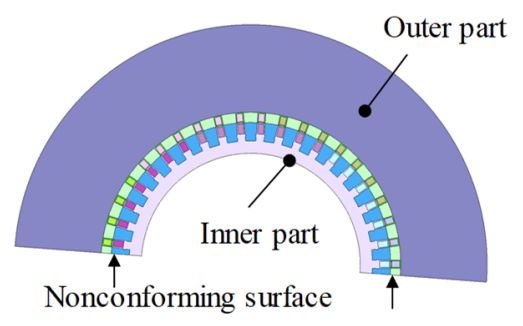

Fig. 6. Base plane of coil end model.

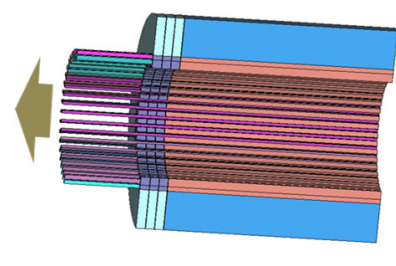

(a) extruded in axial direction

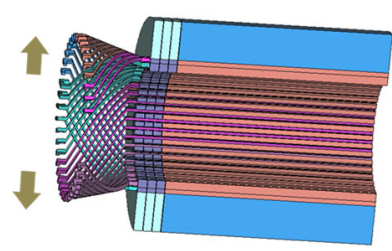

(c) expanded in radial direction

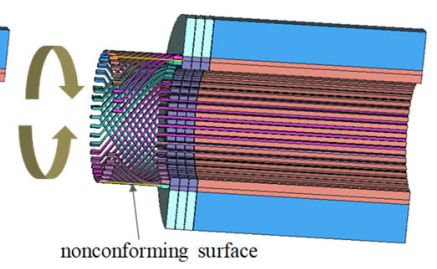

(b) twisted in circumferential direction

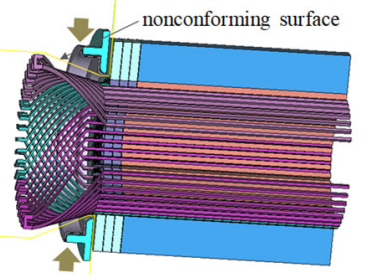

(d) end flange added
Fig. 7. Process of establishing coil end model.

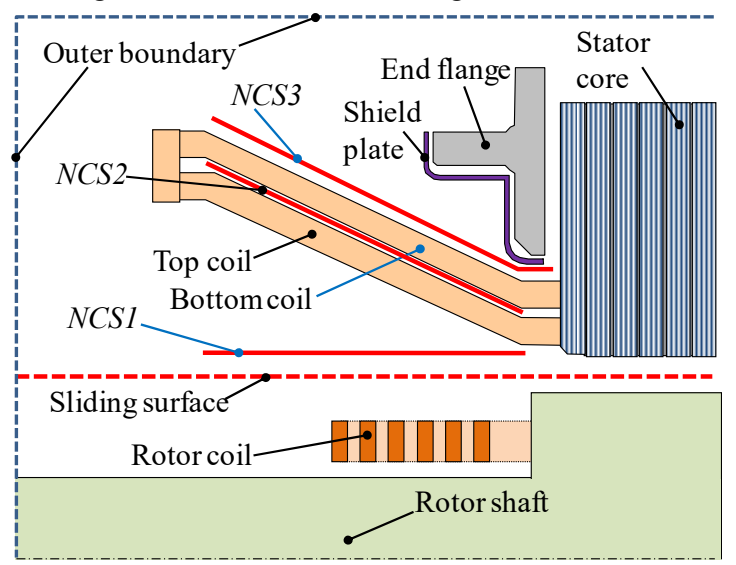

Fig. 8. Cross section and nonconforming mesh connection.

ill distribution of eddy current. Accordingly, the coil end parts can be composed of hexahedral meshes with simple meshing processes.

\subsubsection{Conforming model}

For comparison, an analysis model without nonconforming mesh connection, namely 'conforming model (conf.)', is prepared as shown in Fig. 10. This model was constructed as follows;

I. A base plane mesh of 1-pole region like the mesh in Fig. 5 is created but it is required to consider the outline and the mesh division of the end flange, shield plate and also the coil end part so as to be combined afterwards. For example, the edge lines ' $S$ ' are produced due to the outline of the shield plate. Then, it is extruded in $\mathrm{z}$-direction so as to form a mesh of the stator core region.

II. 2D mesh around the end flange and the shield plate is

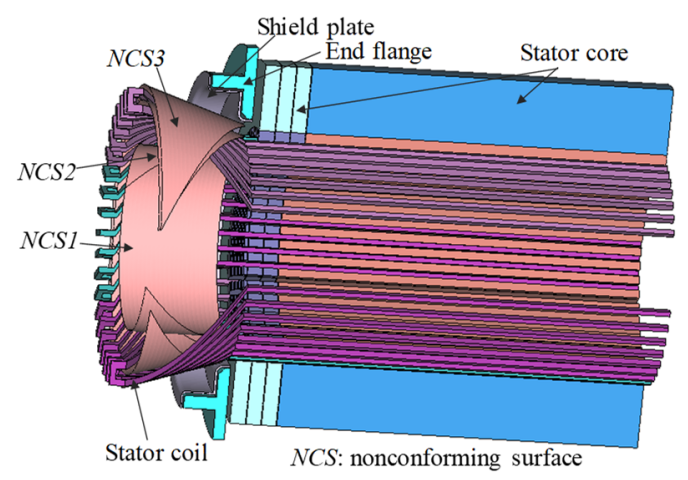

Fig. 9. Stator part and nonconforming surface.

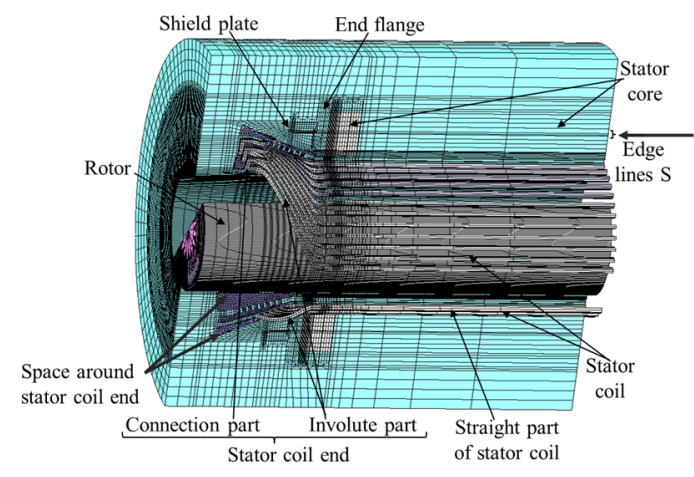

Fig. 10. Coil end model without nonconforming mesh connection (Conforming model).

created in r-z plane and extruded in the $\theta$-direction. As finely divided meshes are required around the surface of the end flange consisting of magnetic conductor, the meshes are connected very carefully with the meshes of other parts because the mesh size of some other parts is not small to avoid increasing the number of meshes.

III. The outlines of the coil end part are drawn with 3D-CAD in consideration of detailed structures such as curves between the involute part and the straight part or the connection part. Though this process might be automated in the design section of manufactures but would take labor for the engineers without automated tools and also in the case of newly developed structures.

IV. The space around the stator coil end is discretized into tetrahedron meshes with auto-meshing method. So the surfaces of the other parts must be discretized with triangleshaped meshes using prism meshes beforehand, otherwise pyramid meshes should be introduced to connect with the hexahedron. This process might need care and attention.

In this way, 'conforming model' is constructed based on more precise shape of the coil end part, considering detailed curves and coil dimension, than the proposed modeling process. But it takes labor and time to create meshes with fewer and well-shaped elements with much care to consider some outlines and mesh division of other parts. The model includes prism meshes in the coil end part, tetrahedral meshes in the air region and pyramid meshes to connect tetrahedral meshes to hexahedral meshes.

\subsubsection{Other conditions}

In both models shown in Fig.9 and Fig. 10., 1/4 region is modeled using boundary conditions and as for the stator core, 3 core blocks 
at the end side are modeled considering the ventilation ducts and the inner part is modeled with the homogenization method ${ }^{(16)}$.

The analysis model parameters such as number of node, element and unknowns are displayed in Table 1 . While the number of elements is almost the same, the number of nodes of the nonconforming model is larger than that of the conforming model. This is because the conforming model was established almost manually and several considerations to reduce element has done and, in addition, this model includes tetrahedral meshes. To complete the conforming model, it took several days, though it may depend on the experience and the circumstances of the engineers, but for the nonconforming model, it took several hours and moreover it could be possible to create meshes within the order of minute by building relatively simple programs because the construction of the mesh is simple.

\section{Analysis results}

5.1 Comparison with measurements Figure 11 shows no-load and short-circuit characteristic curves including the measurements and the analysis results acquired by both the nonconforming and the conforming models. The no-load curve is acquired with the field-current-excited analyses under the opencircuit condition and the induced voltage in the armature windings is examined. The short-circuit curve is obtained with the fieldcurrent-excited analyses in which the circuit is shorted at the terminal. In Fig. 11, 2D analysis results are displayed where the decrease of effective core length due to the ventilation duct is considered by the space factor. Because the end effect is not considered, the induced voltage in the open-circuit condition and the induced current in the short-circuit condition are 3-4\% lower than those measured. This implies the importance of $3 \mathrm{D}$ analysis taking into account the core end and coil end structures. As for the 3D analyses, the difference of the induced voltage in the opencircuit analysis between the nonconforming and the conforming models is below $0.03 \%$ and that of the induced current in the shortcircuit analysis is below $0.2 \%$, which can be regarded as almost the same despite the slight difference of the coil end shape. Then the analysis results of both models show good agreement with the measurements and the differences are approximately under $2 \%$.

5.2 Magnetic flux Figure 12 shows an example of magnetic flux distribution at the cross section of the AA' line calculated with the nonconforming model under the condition of short-circuit operation. The magnetic flux vectors distribute without discontinuity at the nonconforming surfaces.

Figure 13 presents axial magnetic flux distributions impinging on the stator core end. The magnetic flux was examined along the radial direction as the figure depicted in the graph. The expression ' \#3' denotes the tooth at the boundary of the bottom coil phase belt and ' $\# 7$ ' denotes the tooth at the boundary of the top coil phase belt,

Table 1. Analysis Model Parameters of Coil End Model.

\begin{tabular}{|l|c|c|}
\hline & conf. & nonconf. \\
\hline Nodes & 740737 & 962906 \\
\hline Elements & 915743 & 917192 \\
\hline Edges & 2367193 & 2842889 \\
\hline Unknowns & 2334711 & 2306753 \\
\hline Non zeros & 40294807 & 39384865 \\
\hline
\end{tabular}

where the impinging flux is higher than that at other teeth.

For the open-circuit condition, the axial flux distribution at a representative tooth is displayed because it becomes the same at the synchronous operation and the distributions are almost the same for

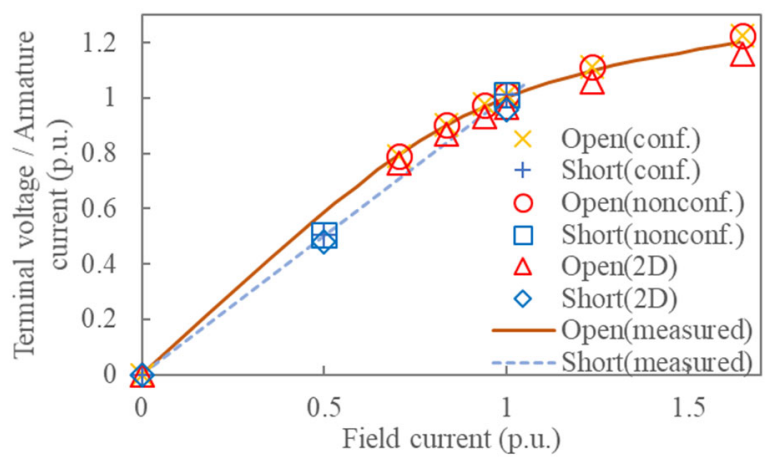

Fig. 11. No-load and short-circuit characteristics curve.

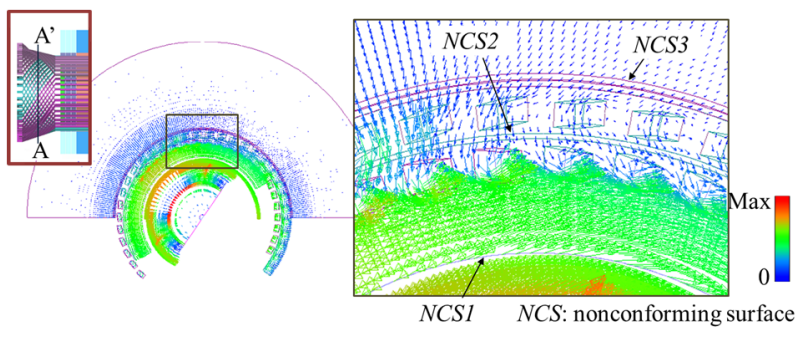

(a) whole view

(b)

enlargement

Fig. 12. Magnetic flux distribution in the cross section indicated as AA'.

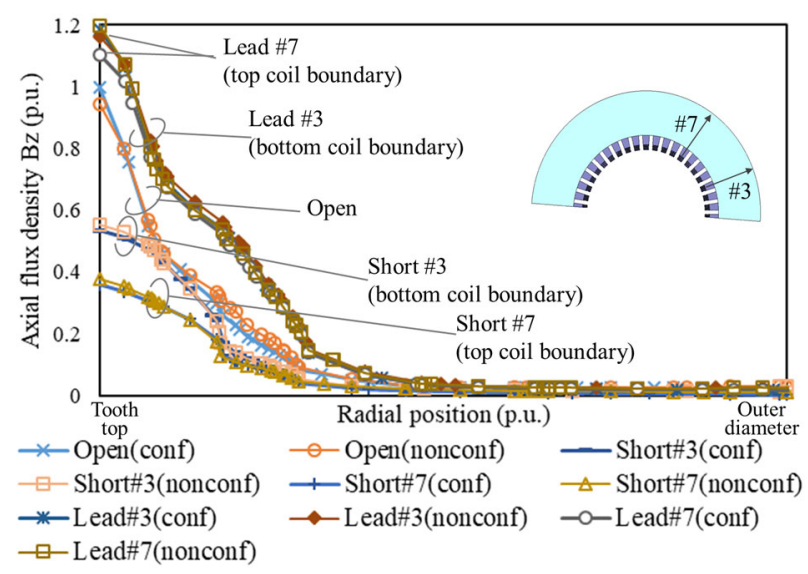

Fig. 13. Axial magnetic flux distribution impinging on the stator core end.

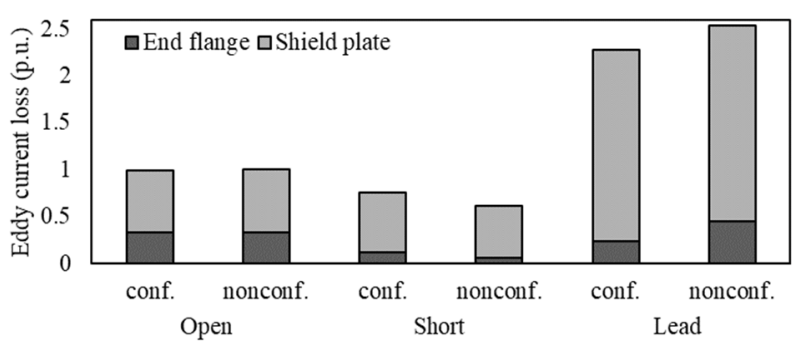

Fig. 14. Eddy current loss in the end flange and the shield plate. 


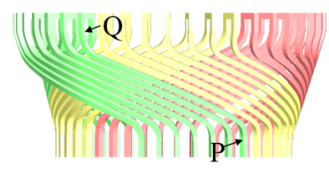

(a) conf

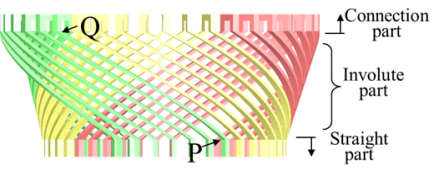

(b) nonconf

Fig. 15. Comparison of model structures around coil end.

the conforming and the nonconforming models.

For the short-circuit condition, there can be seen a difference at the inner region between the third (\#3) and the seventh (\#7) tooth, which is due to the phase location of the armature winding. But the axial flux distributions calculated with the conforming and the nonconforming models agree well.

For the leading load condition, though there is a difference at the seventh tooth tip, the axial flux shows similar distributions at the tooth location for the conforming and nonconforming models.

\subsection{Eddy current loss Figure 14 shows the} comparison of the eddy current loss in the end flange and the shield plate $^{(17)}$. The difference in sum of loss is only $0.3 \%$ for the opencircuit condition where the stator coil current is zero. The difference which is seen under the short-circuit condition and the leading load condition would be due to the difference of the shape of the coil end part. Figure 15 shows the comparison of the model structures around coil end. Whereas the conforming model has curves in the radial and circumferential direction around the boundary of the involute part, the direction of the coil bar of the nonconforming model varies discontinuously at the twisted part so as to connect point $\mathrm{P}$ and $\mathrm{Q}$ in Fig. 15. The actual coil end shape is closer to the conforming model shown in Fig.15 (a).

It could be said that though the eddy current loss in the end flange differs in the coil end shape between the nonconforming and the conforming model, the impinging flux distribution shown in Fig.13 is almost the same. Possible reason is that the magnetic field from the involute part of the coil end has much impact on the eddy current in the end flange and the shield plate, whereas the magnetic field form the straight part of the coil end, of which the difference between the conforming and nonconforming models are small, has impact on the impinging flux on the core end. The magnetic field of the straight part of the coil end would lead to the slot end field which bridges over the core end slots ${ }^{(18)}$. These results may imply that simplification of the coil end shape applied in the nonconforming model would be useful just for evaluate the impinging flux in the core end.

5.4

Calculation time

The comparison of the

Table 2. Comparison of Calculation Time.

\begin{tabular}{|l|c|c|c|c|}
\hline \multirow{2}{*}{} & \multicolumn{2}{|c|}{ conf. } & \multicolumn{2}{c|}{ nonconf. } \\
\cline { 2 - 5 } & open & short & open & short \\
\hline Pre-processing & 0.003 & 0.004 & 0.007 & 0.006 \\
\hline Matrix making & 0.003 & 0.003 & 0.001 & 0.001 \\
\hline Equation solving & 0.988 & 0.722 & 1.449 & 1.332 \\
\hline Post-processing & 0.006 & 0.006 & 0.006 & 0.006 \\
\hline Total & 1.000 & 0.735 & 1.464 & 1.345 \\
\hline Actual time [h] & 4.91 & 3.61 & 7.19 & 6.61 \\
\hline
\end{tabular}

(calculated using a desktop workstation) calculation time is shown in Table 2. These are the time spent for the calculation of the first half cycle. The values are the ratio to the total time of the open-circuit condition of the conforming model except the actual time in the bottom line. The time for solving equation of the nonconforming models is generally longer than that of the conforming models. This is because the matrix condition is worse due to the additional elements for interpolation on the nonconforming surfaces.

\section{Total evaluation of nonconforming mesh connection}

As described in Chapter 5, the calculation time for the nonconforming model tends to become longer than that of the conforming model. But the difference of actual calculation time is about 2.3 hours for a half cycle of the open-circuit condition.

On the other hand, as for the total time including creating the analysis model, it took, for example, several days to complete the mesh of conforming model while several hours for that of the nonconforming model. This difference is much larger than the above-mentioned difference of the calculating time, even considering that several cycles may be required to solve time dependent problems, such as nonlinear eddy current problem.

\section{Conclusion}

In this paper, an electromagnetic analysis method applying the nonconforming mesh connection technique to the FEM is described. The nonconforming mesh connection is expanded from 1D to 2D and the applications to 2 models related to the end region of turbine generators are presented.

In the coil end model, the nonconforming mesh connections are applied to the space around the coil end parts where the top and bottom coils distribute like crossing with grid shape. The results presented good agreement with that of the measurements and also the conforming model for the characteristics curve and the magnetic field distribution. The difference in the eddy current loss would be due to the simplification of the coil end shape.

The method presented in this paper would enable to ease the modeling of the intricately shaped parts in the end region of large rotating machines, which would reduce the modeling time of the engineers.

\section{References}

(1) M.Fujita, T.Sato, N.Takahashi, M.Kobayashi, K.Nagakura, H.Nakamura, H.Kaimori : "Electromagnetic Field Analysis in the End Region of a Large Rotating Machine with Nonconforming Mesh Connection“, Proc. of 23rd Int. Conf. on Electrical Machines and Systems (ICEMS 2020), Hamamatsu (Japan), Nov. (2020)

(2) M.Fujita, T.Ueda, T.Tokumasu, K.Nagakura, M.Kakiuchi, T.Ootaka : "Eddy Current Analysis in the Stator End Structures of Large Capacity Turbine Generators", Proc. of 12th Int. Conf. on Electrical Machines and Systems (ICEMS 2009), Nagasaki (Japan), Nov. (2009)

(3) N.Takahashi, M.Fujita, T.Sato, K.Tsujikawa, K.Nagakura, H.Nakamura, T.Otaka : "Three-dimensional Electromagnetic Analysis of Large Turbine Generator Stator Structures“, IEEJ, RM-17-130 (2017) (in Japanese)

(4) K.Motoyoshi, H.Kometani, N.Sora, S.Maeda : "Large-Scale 3D Electromagnetic Field Analysis for Estimation of Stator End Region Loss in Turbine Generators“, IEEJ Journal of Industry Applications, vol. 6, no. 6, pp.340-345 (2017)

(5) R.Mizokami, M.Muraoka, H.Hiwasa : "Eddy Current Analysis of the Coil End of 2 Pole Turbine Generator“, RM-97-82 (1997) (in Japanese) 
(6) H. Yamashita, A. Yamaji, V. Cingoski, K.Kaneda : "A Novel Tetrahedral Mesh Generation Method for Rotating Machines including End-coil Region“, IEEE Trans. Magn. vol.32, no.3 (1996)

(7) A.Kameari : "Regularization on Ill-posed Source Terms in FEM Computation using Two Magnetic Vector Potentials", IEEE Trans. Magn., vol.40, no.2, pp.1310-1313(2004)

(8) H.Kometani, S.Sakabe, A.Kameari : "3-D Analysis of Induction Motor with Skewed Slots using Regular Coupling Mesh”, IEEE Trans. Magn., vol.36, no.4, pp.1769-1773 (2000)

(9) H.C.Lai, D.Rodger, P.J.Leonald : "Coupling Elements for Problems Involving Movement“, IEEE Trans Magn., vol.28, no.2, pp.1732-1734 (1992)

(10) K.Muramatsu, T.Nakata, N.Takahashi, K.Fujiwara : "3-D Adaptive Mesh Refinement using Nonconforming Elements“, IEEE Trans. Magn., vol.29, no.2, pp.1479-1482 (1993)

(11) C. Golovanov, J. L. Coulomb, Y. Marechal, G. Meunier : "3D mesh connection techniques applied to movement simulation“, IEEE Trans. Magn., vol. 34, no. 5, pp. 3359-3362 (1998)

(12) Y.Okamoto, R.Himeno, K.Ushida, A.Ahagon, K.Fujiwara : "A Dielectric Heating Analysis Method with Accurate Rotational Motion of Stirrer Fan using Nonconforming Mesh Connection“, IEEE Trans. Magn., vol. 44, no. 6, pp.806-809 (2008)

(13) H.Kometani, H.Yamashita : "No-load Iron Loss Analyses for a Turbogenerator", SA-03-70/RM-03-72 (2003) (in Japanese)

(14) A.Kameari : "Three Dimensional Eddy Current Calculation using Edge Elements for Magnetic Vector Potential", Applied Electromagnetics in Materials, Proceedings of the First International Symposium, pp.225-236 (1988)

(15) S.Doi : "The Theory and Calculating Method of Temperature Rise in Armature Core End Constructions of Stream Turbine Generators during Under-excited Operation", Journal of IEEJ, vol. 83, no. 586, pp. 138-146 (1961) (in Japanese)

(16) H.Kaimori, A.Kameari, K.Fujiwara : "FEM Computation of Magnetic Field and Iron Loss in Laminated Iron Core Using Homogenization Method“, IEEE Trans. Magn., vol.43, no.4, pp.1405-1408 (2007)

(17) S. Wakui, K.Takahashi, K.Ide, M.Takahashi, T.Watanabe : "3D Magnetic Field Analysis of a Turbine Generator Stator Core-end Region,", IEEJ Trans. IA, vol. 124, no. 1, pp.77-84 (2004) (in Japanese)

(18) G. Klaus, M. Liese, A.Nakahara, K.Takahashi, K.Ide, K.Hattori, T.Watanabe : "Calculation of losses and temperature rises at the stator core ends of air cooled turbine generators considering the stator slot end field", IEEJ, RM-03-113 (2003).

Masafumi Fujita

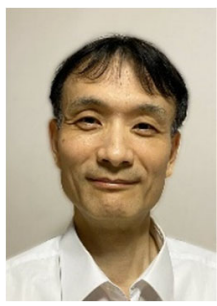

member of the IEEE.

(Senior Member) received the B.E. and M.E. degrees in electrical engineering from Kyoto University, Kyoto, Japan, in 1989 and 1991, respectively, and the Master of Philosophy degree in electrical engineering from University of Bath, Bath, U.K., in 2004. He joined Toshiba Corporation, Yokohama, Japan, in 1991 and has worked on the design and development of electric machines such as rotating machine based on electromagnetic field analysis. Mr. Fujita is a

Takahiro Sato

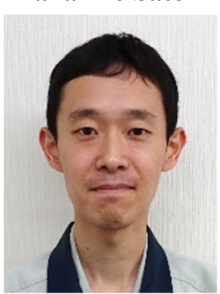

computational electromagnetism, design optimization, and evolutionary algorithms.

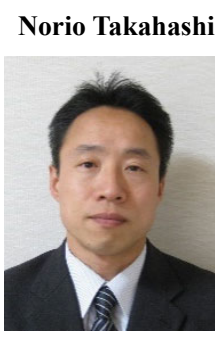

(Member) has worked at Toshiba Corporation from 1992 as a research engineer, and now, he joins Toshiba Energy Systems \& Solutions Corporation. He is engaged in the development of various rotating machinery such as high-speed generators, permanent magnet motors, and turbine generators.

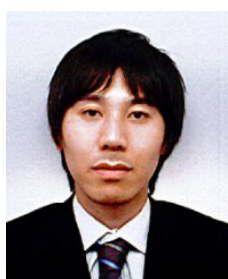

Masashi Kobayashi (Member) received the M.E. degree in engineering from Tokyo Institute of Technology, Japan, in 2008. Since 2008, he has worked as an engineer at Toshi ba Corporation, and now, he joins Toshiba Energy Systems \& Solutions Corporation. $\mathrm{He}$ is engaged in the development of various turbine generators.
Ken Nagakura

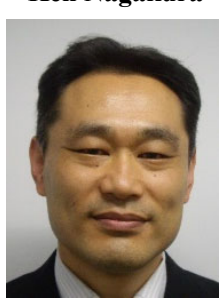

(Member) received the M.E. degree in engineering from Keio University, Japan, in 1998. Since 1998, he has worked as an engineer at Toshiba Corporation, and now, he joins Toshiba Energy Systems \& Solutions Corporation. He has been engaged in the development of various turbine generators.
Hideyuki Nakamura (Member) received the B.S. degree in engineering

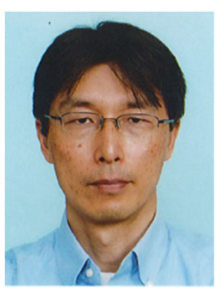
from University of Tokyo, Japan, in 1988. Since 1988, he has worked as an engineer at Toshiba Corporation, and now, he joins Toshiba Energy Systems \& Solutions Corporation. $\mathrm{He}$ is engaged in the development of various turbine generators.

Hiroyuki Kaimori (Member) received the M.S. degree from Toyo

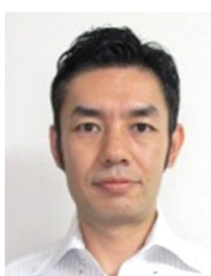
University, Japan, in 2002. Since April 2002, he joins at Science Solutions International Laboratory, Inc., Japan. He works for electromagnetic simulation software as a developer. His research interests are electromagnetic analysis and coupling analysis with circuit simulation of electrical machines. Mr. Kaimori is a member of IEEE. 